\title{
Bridge Topology Optimisation with Stress, Displacement and Frequency Constraints
}

\author{
Hong Guan*, Yin-Jung Chen, Yew-Chaye Loo \\ School of Engineering, Griffith University Gold Coast Campus, PMB 50 Gold Coast Mail Centre, \\ Queensland 9726, Australia
}

Yi-Min Xie

School of Civil and Chemical Engineering, Royal Melbourne Institute of Technology, City Campus, GPO Box 2476V, Melbourne 3001, Australia

Grant P. Steven

School of Engineering, University of Durham, Durham, DH1 3LE, UK

\begin{abstract}
The principal stress based Evolutionary Structural Optimisation (ESO) method is presented herein for topology optimisation of arch, tied arch, cable-stayed and suspension bridges with both stress and displacement constraints. Two performance index formulas are developed to determine the efficiency of the topology design. A refined mesh scheme is proposed to improve the details of the final topology without resorting to the complete analysis of a finer mesh. Furthermore, cable-supported bridges are optimised with frequency constraint incorporating the "nibbling" technique. The applicability, simplicity and effectiveness of the method are demonstrated through the topology optimisation of the four types of bridges.
\end{abstract}

Key words: topology optimisation, bridge structures, design constraints

\section{Introduction}

Bridges are remarkable, eye-catching structures which are often regarded as landmarks. Traditionally, bridge structures are designed based on engineering theories and

\footnotetext{
* Corresponding author. Tel: +61-7-5552-8708; fax: +61-7-5552 8065.

E-mail address: H.Guan@mailbox.gu.edu.au (H. Guan).
} 
previous experience, which would involve the preliminary design, structural analysis and check against strength/stiffness/stability/frequency requirements. This is followed by design modification, re-analysis and re-checking. Undoubtedly, such design process is very expensive and time-consuming. With the rapid development of advanced computer technologies, sophisticated optimum design approaches have gained increasing popularity in recent years as they can significantly improve the efficiency of a design. According to the prescribed structural type, loading, material and code-specified requirements, an optimum design defines a set of design variables and constraints as well as the target function. It involves a loop of structural analysis, structural optimisation, re-analysis and reoptimisation. A rational distribution of the material in a structure can only be achieved by the optimum design. For this reason, a safe and economic structural design is ensured.

The aim of topology optimisation is to find a conceptual layout of a design by distributing a given amount of material in a domain thereby achieving the lightest and stiffest structure while satisfying certain specified design constraints. Unlike the conventional shape optimisation, topology optimisation does not generate the optimum shape from an initial known shape. Topology optimisation is of considerable practical interest due to the fact that it can achieve much greater savings and much increased system performances than the mere cross-section (sizing) optimisation. Topology optimisation can not only improve significantly the efficiency of the design, but also serve as a preprocessing tool for detailed sizing and shape optimisation.

Extensive research effort has been made in the area of topology optimisation of both discrete and continuum structures, as summarised by Bendsøe and Kikuchi [1], Topping [2], Rozvany et al. [3] and Thierauf [4]. A classical truss topology optimisation problem is to achieve minimum weight subject to equilibrium and stress constraints. Such problem can conveniently be formulated in terms of the ground structure approach where the optimal substructures can be found from a set of all possible connected bars defined on a discrete grid (see Ref. [5]). The method of (discretized) optimality criteria, proposed by Rozvany et al. (see Refs. [6,7]), was derived by applying the duality theory of mathematical programming to a separable approximation of the design problem. It has been found particularly efficient for the topology optimisation of multipurpose structures (see Ref. [8]). For topology optimisation of continuum structures, Bendsøe and Kikuchi [9], Suzuki and Kikuchi [10], and Bendsøe et al. [11] developed a homogenisation method based on the 
homogenisation theory where the initial design domain is homogeneous at the macroscopic scale. The method has been successfully applied to solve many types of topology optimisation problems including composite material structures (see Ref. [12]) and those with multiple constraints (see Ref. [13]). Another distinct approach to topology optimisation of continuum structures is the density method (see Ref. [14]) where a simple energy method is used and a formula for Young's modulus is assumed thereby generating optimal material distribution. More recently, stochastic algorithms such as genetic algorithms have received increasing attention. Genetic algorithms which follow a global search procedure are capable of gradually improving the solution in succeeding populations using operations that mimic those of the natural evolution (see Ref. [15]). However such random search methods require a great number of function evaluations and are very expensive in terms of computational cost. Rozvany [8], in discussing the efficiency and robustness of various optimisation methods, states that the optimality criteria methods are most efficient but least robust whereas the random search methods are least efficient but most robust. The writer also comments that the Evolutionary Structural Optimisation (ESO) method proposed by Xie and Steven (see Refs. [16,17]) and the fully stressed design method by Hinton and Sienz (see Ref. [18]) have shown to be very efficient in achieving good practical solutions.

Compared to other methods for structural optimisation, the ESO method is attractive due to its simplicity in concept and effectiveness in application. The conventional ESO method employing the von Mises stress $\left(\sigma_{V M}\right)$ as the optimisation criterion, has a simple concept of producing a fully stressed design by systematically removing inefficient material from an oversized structure (see Ref. [16]). The method has proven to be successful in solving practical structural problems. However, it cannot be used to produce topologies for various bridge type structures with complicated geometry and loading conditions (see Ref. [19]) because it does not take into account the actual material properties in tension and compression. Most engineering structures including bridges are constructed of materials like concrete which are strong in compression, or of materials like steel which are highly effective in tension. Examples of such structures include arch bridges where every part of the arch is under compression and cable-stayed bridges where the cables work solely in tension. To optimise such material-oriented structures, the characteristics of different materials and their tension and compression behaviour must be monitored. This has led to the present principal stress based ESO method (see Refs. [20,21]) which is employed in this study to directly obtain the most appropriate bridge topology. The methodology, in 
conjunction with the stress, displacement and frequency considerations, is discussed in some details. The applicability, simplicity and effectiveness of the method are confirmed through the topology optimisation of various bridges.

\section{Principal stress based ESO method}

To achieve optimum design of engineering structures that makes the best utilisation of the available materials, the conventional ESO method has been extended to cover tensionand compression-dominant designs (see Ref. [20]). This is accomplished by introducing into the optimisation process the principal stress criteria or the maximum and the minimum principal stresses $\left(\sigma_{11}\right.$ and $\left.\sigma_{22}\right)$. Relevant design constraints are described in Section 2.1 followed by the derivation of the performance index formulas for design efficiency measurement.

\subsection{Design constraints for tension/compression-dominant designs}

\subsubsection{Stress constraint}

Two parallel algorithms were developed by Guan et al. to differentiate tension- and compression-oriented elements in a design area based on which the tension- or compressiondominant design with stress constraint can be performed [21]. Similar to the conventional ESO method, a linear static analysis is carried out on a large enough design domain with fine mesh of finite elements. Consequently, each element's maximum and minimum principal stresses $\sigma_{11}^{e}$ and $\sigma_{22}^{e}$ can be determined. If a tension-dominant structure is to be designed, the compression-oriented elements (whose $\sigma_{22} \leq 0.0$ and $\left|\sigma_{22}\right|>>\left|\sigma_{11}\right|$ ) should be removed. On the contrary, if a compression-dominant structure is desired, the tension-oriented elements (whose $\sigma_{11} \geq 0.0$ and $\left|\sigma_{11}\right|>>\left|\sigma_{22}\right|$ ) should be eliminated. This has led to the new element removal algorithms which form the core of the principal stress based ESO method. In a mathematical form, to generate a tension-dominant structure, remove the elements if

$$
\left|\sigma_{11}^{e}\right| \leq R R_{i} \times\left|\sigma_{11, \max }\right| \quad \text { when } \quad \sigma_{22}^{e} \leq 0.0
$$


And, to generate a compression-dominant structure, remove the elements if

$$
\left|\sigma_{22}^{e}\right| \leq R R_{i} \times\left|\sigma_{22, \max }\right| \quad \text { when } \quad \sigma_{11}^{e} \geq 0.0
$$

where $\left|\sigma_{11, \max }\right|$ is the absolute maximum value of $\sigma_{11}$ among the elements whose $\sigma_{22}^{e} \leq 0.0$; $\left|\sigma_{22, \text { max }}\right|$ is the absolute maximum value of $\sigma_{22}$ among the elements whose $\sigma_{11}^{e} \geq 0.0 ; R R_{i}$ is the rejection ratio to ensure that only a small number of elements are removed each time. When considering the stress constraint only, an iterative procedure is performed which is defined by repeated cycles of finite element analysis and systematic removal of inefficiently used material. Each cycle is continued using the same value of $R R_{i}$, until no more elements are deleted at the current iteration $i$. To proceed to the next iteration, $R R_{i}$ has to be increased by adding the evolution ratio ER. Such a process continues until a desired topology of improved quality is obtained.

Note that when the structure is subjected to multiple load cases, an element can be removed only if Eq. (1) or (2) is satisfied by all the load cases present in the structure. This is to ensure that the optimum design is achieved in which every part of the remaining material has its own role to play for at least one load case and possibly for all load cases.

\subsubsection{Stress and displacement constraints}

With stress constraint only, the principal stress based ESO has provided an effective means of generating tension- and compression-dominant designs (see Ref. [20]). However, even with its extended capability in comparison with the conventional ESO method, the principal stress based ESO still features a stress driven optimisation technique. In an engineering sense, it is more rigorous to optimise a structure with both stress and displacement constraints. In the light of this, the displacement control must be imposed in addition to removing the elements that satisfy the stress condition. This requires the determination of the displacement sensitivity number $\alpha_{d, i}$ based on the formulas developed by Xie and Steven [17]. 
In a finite element analysis, the static behaviour of a structure can be expressed by the stiffness equation as

$$
\boldsymbol{K} \cdot \boldsymbol{u}=\boldsymbol{P}
$$

where $\boldsymbol{K}$ is the global stiffness matrix of a structure and, $\boldsymbol{u}$ and $\boldsymbol{P}$ are, respectively, the global nodal displacement and nodal load vectors. Assuming that the element $i(i=1, N)$ is to be removed from a structure where $N$ is the total number of elements in the design domain. This would induce a change in the stiffness matrix, $\Delta \boldsymbol{K}_{i}$, as well as a change in the displacement vector $\Delta \boldsymbol{u}$. However, it is assumed that the element removal has no effect on the load vector. By manipulating the modified stiffness equation, the change in the specified $j$ th displacement component $u_{j}$ due to the removal of the ith element can be represented by the displacement sensitivity number $\alpha_{d, i}$. Or,

$$
\alpha_{d, i}=\left|\alpha_{d, i j}\right|=\left|\boldsymbol{u}_{i j}^{\mathrm{T}} \cdot \boldsymbol{K}_{i} \cdot \mathbf{u}_{i}\right|
$$

where $\alpha_{d, i j}$ can be a positive or negative quantity. Note that $u_{j}$ is to be limited to a prescribed value, $u_{j}^{*}$, in the design process (i.e. $\left|u_{j}\right| \leq u_{j}^{*}$ ). Also in Eq. (4), $\boldsymbol{u}_{i}$ and $\boldsymbol{u}_{i j}$ are respectively the displacement vectors of the ith element due to the real load $\boldsymbol{P}$ and due to the virtual unit load $\boldsymbol{F}_{j} ; \boldsymbol{K}_{i}$ is the stiffness matrix of the ith element and is equal but opposite to $\Delta \boldsymbol{K}_{i}$. In a more general case when a structure is designed for multiple load cases $\boldsymbol{P}_{k}(k=1$, $L)$ and is subjected to multiple displacement constraints $u_{j k}^{*}(j=1, M)$, the sensitivity number can then be derived as

$$
\alpha_{d, i}=\sum_{k=1}^{L} \sum_{j=1}^{M} \lambda_{j k} \cdot\left|\alpha_{d, i j k}\right|=\sum_{k=1}^{L} \sum_{j=1}^{M} \lambda_{j k} \cdot\left|\boldsymbol{u}_{i j}^{T} \cdot \boldsymbol{K}_{i} \cdot \boldsymbol{u}_{i k}\right| \quad(i=1, N)
$$

in which $\boldsymbol{u}_{i k}$ is the displacement vector of the ith element due to load case $\boldsymbol{P}_{k} ; L$ and $M$ are, respectively, the total number of load cases and that of the displacement constraints. In Eq. (5), $\lambda_{j k}=\left|u_{j k}\right| / u_{j k}^{*}$ is the weighting parameter indicating the contribution of the $j$ th displacement constraint under the $k$ th load case. 
To minimise the displacement change $\left|u_{j k}\right|$ during the optimisation process, the elements that have the lowest $\alpha_{d, i}$ among all or those whose $\alpha_{d, i j k}$ are closest to zero should be removed from the design domain. The proposed optimisation procedure considering both stress and displacement constraints may be summarised as follows:

1. Discretize a large enough area that can cover the final design into a dense finite element mesh.

2. Define a range variable $(r)$ to be used with the displacement constraint.

3. Specify the direction and location of the displacement constraint as well as the value of the imposed displacement limit.

4. Solve the stiffness equation for each of the actual loads $\boldsymbol{P}_{k}(k=1, L)$ and for the virtual unit load $\boldsymbol{F}$.

5. Determine the number of elements $\left(N_{s}\right)$ with the lowest principal stress $\left|\sigma_{11}^{e}\right|$ or $\left|\sigma_{22}^{e}\right|$, for tension- or compression-dominant design, respectively, according to Eq. (1) or (2).

6. Calculate the sensitivity number $\alpha_{d, i}$ using Eq. (5) for each of the elements $\left(N_{s}\right.$ in total) that satisfies the stress condition.

7. Remove a number of elements $\left(N_{d}\right)$ with the lowest $\alpha_{d, i}$ where $\left(N_{d}\right)$ is equal to the range variable ( $r$ ). Note that $N_{d} \subseteq N_{s}$.

8. Repeat Steps 4 to 7 until the displacement limit is reached.

The above element removal scheme implies that whilst the lowly stressed element is removed from the structure, the remaining part of the structure is still stiff enough and its maximum deflection must not exceed the prescribed limit. This scheme is employed to optimise an over designed domain until a reasonable topology is generated that satisfy both stress and displacement conditions.

\subsubsection{Frequency constraint with "nibbling" technique}

The optimum design of structures satisfying frequency constraint is also of great importance, particularly for bridge type structures. It is often necessary to manipulate the natural frequencies of a structure away from the frequency range of dynamic excitation in 
order to avoid resonant conditions and to prevent induced damage to the structure. In a finite element analysis, the state of free vibrations of a structure is governed by the eigenvalue problem as

$$
\left(\boldsymbol{K}-\omega_{n}^{2} \cdot \boldsymbol{M}\right) \cdot \boldsymbol{u}_{n}=0
$$

where $\boldsymbol{K}$ and $\boldsymbol{M}$ are respectively the global stiffness and mass matrices; $\omega_{n}$ is the $n$th natural frequency; $\boldsymbol{u}_{n}$ is the $n$th eigenvector.

The change in frequency $\omega_{n}^{2}$ due to the removal of the ith element can be derived based on the Rayleigh concept and the assumption of insignificant variation in mode shape (see Ref. [17]). This leads to the frequency sensitivity number $\alpha_{f, i}$, as an indicator of the change in $\omega_{n}^{2}$. Or,

$$
\alpha_{f, i}=\boldsymbol{u}_{n, i}^{\mathrm{T}} \cdot\left(\omega_{i}^{2} \cdot \boldsymbol{M}_{i}-\boldsymbol{K}_{i}\right) \cdot \boldsymbol{u}_{n, i}
$$

where $\boldsymbol{K}_{i}$ and $\boldsymbol{M}_{i}$ are respectively the stiffness and mass matrices of the ith element; $\boldsymbol{u}_{n, i}$ are the components of the $n$th structural eigenvector of the ith element and is normalized with respect to $\boldsymbol{M}$. Note that $\alpha_{f, i}$ can be positive or negative. To increase a specified frequency $\omega_{n}$, the elements that have the maximum positive $\alpha_{f, i}$ should be removed from the design domain.

An optimisation scheme considering frequency constraint is proposed herein. The frequency analysis is performed on a resulting topology (close to the optimum design) generated with the stress and displacement constraints. Aiming at improving the details of the resulting topology while increasing the fundamental frequency of the structure, the frequency optimisation is performed without creating further cavities in the remaining material. This requires the use of a "nibbling" technique proposed by Xie and Steven [17] where only the surrounding shape/perimeter of the topology is altered. The optimisation procedure incorporating the frequency constraint with "nibbling" technique is summarised below in 5 steps: 
1. Select the appropriate topology that is generated with the stress and displacement constraints.

2. Solve the eigenvalue problem using Eq. (6).

3. Calculate the sensitivity number $\alpha_{f, i}$ for each element according to Eq. (7).

4. To increase the frequency, remove no more than 10 elements of the maximum positive $\alpha_{f, i}$. Note that the elements to be removed must also satisfy the condition that at least one of the element's edges or sides is not connected to any other elements in the structure. This is to ensure that only the structural boundaries are modified without creating holes in the remaining material.

5. Repeat Steps 2 to 4 until an optimum design is reached when no more elements can be removed.

The proposed element removal scheme incorporating the frequency constraint with "nibbling" technique is capable of increasing the fundamental frequency of the structure and improving the details of the final topology. In other words, the topology can be re-shaped under the frequency constraint following the optimisation with stress and displacement constraints.

Note that the current investigation deals with element removal only where removed elements no longer exist in the finite element grid. It is recognised that a bi-directional ESO method (BESO) based on the process of adding and removing elements has become available (see Ref. [22]). The BESO methos involves setting up a reasonable initial design, which is large enough to allow unnecessary materials to be removed. Such process can be tedious for practical structures like bridges with complicated geometry and loading conditions.

\subsection{Performance index formulas}

One of the critical concerns in topology optimisation is the determination of the efficiency of a series of designs generated during the optimisation process. In other words, an indicator is essential to compare the performances of the progressive designs and from which, an optimum topology can be determined. A performance index $(P I)$ in terms of von Mises stress has been proposed by Liang et al. [23] based on a scaling design concept by 
Kirsch [24] where the actual design variable such as the element thickness is scaled with respect to the design constraint. Based on the same scaling design concept, two PI formulas associated with the tension-/compression-dominant design are developed herein to cover the situation where the principal stresses are used as the optimisation criteria.

For tension-/compression-dominant design, the optimization of the continuum structures can be posed in the following form:

$$
\begin{array}{ll}
\text { minimize } & V=\sum_{e=1}^{N} V_{e} \\
\text { subject to } & \left|\sigma_{22, \text { max }}\right| \leq\left|\sigma_{22}^{*}\right| \quad \text { for tension-dominant design } \\
\text { or } & \left|\sigma_{11, \text { max }}\right| \leq\left|\sigma_{11}^{*}\right| \quad \text { for compression-dominant design }
\end{array}
$$

where $V$ and $V_{e}$ are respectively the volume of the total material and that of any individual element $e ; \sigma_{11}^{*}$ (or $\sigma_{22}^{*}$ ) is the maximum (or minimum) principal stress limit. The stress constraint given in Eq. (9) implies that for tension-dominant design, the absolute maximum value of $\sigma_{22}$ in the design domain must not exceed the prescribed stress limit $\sigma_{22}^{*}$. For compression-dominant design, on the other hand, the maximum $\sigma_{11}$ in the design domain must not exceed the prescribed stress limit $\sigma_{11}^{*}$.

For the linear elastic plane stress problems, the structural stiffness matrix is a linear function of the design variable such as the thickness or the volume of the structure. For a bridge structure under consideration, each structural component, of different cross-sectional thickness, can be assigned design or non-design domains. The non-design domain is normally defined for support regions and for areas where the structural form of certain components is known a priori. The material in the non-design domain is not removable during the entire optimisation process. This results in the volume variation in the design domain only. Hence, it is more meaningful and accurate to consider the stress and the volume of the design domain. Such consideration is reflected by a subscript "d" in the PI formulas. 
When tension-dominant design is performed, the volume of the design domain can be scaled with respect to the stress constraint. As a result, the relative volume of the initial (original) design domain, $V_{d, o}^{\prime}$, can be expressed as

$$
V_{d, o}^{\prime}=V_{d, o} \cdot\left(\left|\sigma_{22, \max }\right|_{d, o} /\left|\sigma_{22}^{*}\right|\right)
$$

in which $V_{d, o}$ and $\left|\sigma_{22, \max }\right|_{d, o}$ are respectively the volume of, and the $\left|\sigma_{22, \max }\right|$, in the original design domain. In an iterative optimisation process, the relative volume of the current design at ith iteration, $V_{d, i}^{\prime}$, can also be scaled as

$$
V_{d, i}^{\prime}=V_{d, i} \cdot\left(\left|\sigma_{22, \max }\right|_{d, i} /\left|\sigma_{22}^{*}\right|\right)
$$

where $V_{d, i}$ and $\left|\sigma_{22, \max }\right|_{d, i}$ are respectively the volume of, and the $\left|\sigma_{22, \max }\right|$, in the current design domain at ith iteration.

The performance index $P I_{d, t}$ at the ith iteration can then be determined as

$$
P I_{d, t}=\frac{V_{d, o}^{\prime}}{V_{d, i}^{\prime}}=\frac{\left|\sigma_{22, \max }\right|_{d, o} \cdot V_{d, o}}{\left|\sigma_{22, \max }\right|_{d, i} \cdot V_{d, i}}
$$

Similarly for compression-dominant design,

$$
P I_{d, c}=\frac{V_{d, o}^{\prime}}{V_{d, i}^{\prime}}=\frac{\left|\sigma_{11, \max }\right|_{d, o} \cdot V_{d, o}}{\left|\sigma_{11, \max }\right|_{d, i} \cdot V_{d, i}}
$$

where $\left|\sigma_{11, \max }\right|_{d, o}$ and $\left|\sigma_{11, \max }\right|_{d, i}$ are respectively the $\left|\sigma_{11, \max }\right|$ in the design domain at the initial stage and at the ith iteration. 
With the performance index formulas in hand, the efficiency of the material-oriented topology designs can be measured during the optimisation process. The Performance Index $P I_{d, t}$ (or $P I_{d, c}$ ) can also reflect the changes in the volume and the principal stress level in the design domain. Note that $P I_{d, t}$ and $P I_{d, c}$ are dimensionless quantities and they increase with the optimisation process, which suggests that the topology of the structure is improved by systematically removing under-utilised material. The optimum topology can then be selected as the one corresponding to the highest $P I_{d, t}$ (or $P I_{d, c}$ ) value. It should be noted that there may exist several local maxima on the PI curve, which cannot be considered as an optimum because the prescribed displacement limit is not reached.

\section{Optimisation of bridges with stress and displacement constraints}

To illustrate the capability of the principal stress based ESO method in optimising tension and compression-dominant designs, four bridge type structures are studied with both stress and displacement constraints. They are the arch, tied arch, cable-stayed and suspension bridges. These structures are considered to be the most amenable design models as they have distinct characteristics in structural forms and they are constructed with major or dominant tension and/or compression components. Note that the main objective of this study is to produce optimum topology for either compression- or tension-dominant structural components, therefore for arch and tied-arch bridges, the focus is to optimise the arch profile; whereas for cable-stayed and suspension bridges, generate optimum outlines of cable(s).

\subsection{Refined mesh scheme}

Some research attempts have been made in the past two years in the optimisation of bridge structures with the same (or varying) material properties and uniform (or nonuniform) thickness for the design and non-design domains (see Ref. [25]). The results have confirmed the applicability and effectiveness of the principal stress based ESO in generating tension- and compression-dominant designs of bridge structures. The optimum topologies obtained with the stress and displacement constraints well resemble, to a certain extent, the actual outlines of the bridges. However, due to the limited number of elements adopted in the analysis, the details of the design topologies were some way from perfection particularly for tied arch, cable-stayed and suspension bridges. It was obvious that an improved design 
necessitated a much finer mesh. This unfortunately would significantly increase the computational cost. In the light of this, a refined mesh scheme is proposed herein to further improve the final design that is already produced using a regular mesh. This scheme aims at generating the optimum topologies more economically without resorting to a complete, but costly analysis of a finer mesh. A detailed implementation of the scheme is presented in the next section.

\section{2. $\quad$ Bridge material properties and load arrangements}

All the bridge dimensions are quoted from existing bridges (see Refs. [26,27]). The material properties and loadings are also determined using the appropriate values recommended for bridge design. This is to ensure that the optimum designs can better reflect the real world situation. The appropriate values of the displacement limit are used for each type of bridges. These values are determined based on the recommendation of the Australian Bridge Design Code (see Ref. [28]) where the deflection allowance under the service load should not exceed 1/800 of the main span of the bridge. The bridges are constructed using concrete and/or steel depending on their structural types and functionalities. For concrete, the modulus of elasticity $E$ is 21,000 MPa, the material density $\rho$ is $2400 \mathrm{~kg} / \mathrm{m}^{3}$ and the Poisson's ratio $v$ is 0.2 . For steel, $E$ is $210 \mathrm{GPa}, \rho$ is $7800 \mathrm{~kg} / \mathrm{m}^{3}$ and $v$ is 0.3 . As the analysis is based on the plane stress assumption, the equivalent crosssectional thickness of three-dimensional structures is adopted. In all the analyses, the rejection ratio $R R_{i}$ and the evolution ratio $E R$ are both taken as 0.1 .

All the bridges are investigated under two loading conditions, viz, the uniformly distributed load (UDL) and the combined UDL and moving loads. When the design domain is subjected to the UDL, only half the domain is considered due to symmetry. Under such loading condition, it is necessary to consider two load cases: one is the UDL (100 kN/m); the other is the unit virtual load applied at the mid-span of the top of the deck where the displacement is to be controlled. For the combined loading condition, the entire domain with ten load cases is considered. Each of the first nine load cases is the combination of a UDL $(12.5 \mathrm{kN} / \mathrm{m})$ and a moving point load $(200 \mathrm{kN})$. The UDL on the top of the bridge deck is to simulate the service load while the moving point load acting consecutively from left to right across the bridge (at nine equally spaced locations) is to simulate the traffic load. 
Load case 10, at the mid-span and on the top of the deck, is a unit virtual load for the displacement control.

\subsubsection{Arch bridge}

The dimension of the initial design domain for the entire bridge is $210 \mathrm{~m} \times 21 \mathrm{~m}$, a symmetrical half of which is presented in Fig. 1 under the UDL condition, whereas Fig. 2 shows the full domain (with $180 \times 18$ four-node square elements) under the combined loading. The bridge deck and the abutments are assumed to be non-design domains and are represented by the shaded elements. The entire arch bridge, of concrete, has uniform thickness of $8 \mathrm{~m}$ in cross-section. The thickness input is required when self-weight of a structure is considered which has been reported by Chen [25]. To provide adequate support both vertically and horizontally at the springings of the arch, the bottom of the left and right regions of the design domain are fixed to the ground. Note in Fig. 1(a) that load case 1 contains the UDL of $100 \mathrm{kN} / \mathrm{m}$ on the top of the deck and a fictitious load of $0.0001 \mathrm{kN}$ at the extreme support locations. The application of such fictitious load is to generate a point load file that is required by the optimisation program. The prescribed mid-span displacement limit for both loading conditions is $0.1 \mathrm{~m}$. The optimisation process is performed using the compression-dominant design algorithm and the $P I_{d, c}$ formula (given in Eq. (13)) is used for the selection of the optimum topology.

Figs. 3(a) and (b) show, respectively, the optimisation histories of the arch bridge under UDL and the combined loading. They are represented by the performance index $\left(P I_{d, c}\right)$ and the volume ratio $\left(V_{d, i} / V_{d, o}\right)$ against the iteration number. As can be seen in the figures, the $P I_{d, c}$ values show a tendency to increase. At the first iteration, $P I_{d, c}$ is equal to 1 when no elements are removed. By gradually removing the lowly stressed elements from the initial design domain, the stress levels of the remaining elements become more uniform. Note that the $P I_{d, c}$ curves exhibit sudden jumps at some stages. This is due to a large reduction in the stress level. The volume of the remaining material decreases gradually with the increase in $P I_{d, c}$ values.

The maximum $P I_{d, c}$ values are reached at iterations 66 and 122, respectively, for the two loading conditions where the corresponding volume reductions are $60 \%$ and $71 \%$. The optimization history suggests that a least weight and more uniformly stressed design has 
been achieved. The corresponding optimum topologies that are generated at the maximum $P I_{d, c}$ values are presented in Fig. 4. For both loading conditions, a prominent arch rib - a load-bearing component in compression is generated. This confirms the natural strength of the arch bridge where its total weight is carried outward along the curve of the arch. Also formed in both topologies are two additional inclined members at each side of the arch rib. Such small components are there to transfer the applied loads to the load-carrying part of the bridge. In general, the optimum topologies well resemble that of the actual arch bridge (see Ref. [26]).

\subsubsection{Tied arch bridge}

The initial design domain for the entire bridge, of dimension $330 \mathrm{~m} \times 55 \mathrm{~m}$, is discretized into $138 \times 23$ square elements for the combined loading condition, as shown in Fig. 5(a) under the first nine load cases. Load case 10 is the unit virtual load at the mid-span location on top of the deck. Again half the design domain is analysed when the bridge is under UDL. As can be seen in Fig. 5(a), the hangers are assumed to be equally spaced; all the hangers and the bridge deck are assumed to be non-design domains. To simulate the abutments that can provide axial thrust to the arch rib, the two ends of the bridge deck are fixed. The thickness of the concrete deck is $14 \mathrm{~m}$. The non-design hangers and the design domain for arch region have steel material properties and their element thicknesses are 3.1 $\mathrm{m}$. The prescribed mid-span displacement limit is $0.15 \mathrm{~m}$. The compression-dominant design algorithm is adopted and the optimum topology can be identified by comparing $P I_{d, c}$ during the optimisation process.

To improve the final design, the refined mesh scheme is introduced. It involves the optimisation of a regular mesh followed by that of a refined mesh. The regular mesh scheme is based on the initial design domain with fine mesh (Fig. 5(a)). When the initial design domain evolves to an intermediate stage where a stress-oriented domain starts to form, the original regular mesh is further refined. Subsequently, the design of a refined mesh commences and the optimisation process continues.

For the tied arch bridge under combined loading condition, the suitable mesh for refinement is selected based on the observation of the $P I_{d, c}$ curve presented in Fig. 6 . It can 
be seen that the $P I_{d, c}$ curve exhibits a sudden jump at iteration 97. At the corresponding iteration, or the intermediate stage when a possible area of arch starts to form, the regular mesh is backed up and each of its elements is subdivided into 4 smaller elements, thereby generating a refined mesh as shown in Fig. 5(b). The optimisation process based on the refined mesh is hence continued. This is also reflected in the optimisation history plots (Fig. 6) where the dash line indicates the transition from the regular to the refined mesh schemes, and the dash-dot line marks the iteration when the optimum design is achieved under the refined mesh scheme. It can be seen in Fig. 6 that the $P I_{d, c}$ peaks at iteration 167 which indicates that the optimum topology is produced. Fig. 7 shows the optimum topologies of the bridge under the two loading conditions. The slight difference between the two topologies shown in Figs. 7(a) and 7(b) is believed to be affected by the closely spaced hangers. In view of this, the positions and the amount of hangers may be varied to examine their influence on the optimum topologies.

In comparison with the optimum topologies generated with a regular mesh (see Ref. [25]), the current design with the proposed refined mesh scheme produces a reduced depth of arch and a much smoother outline. In addition, more under-utilised material is removed i.e. with 73\% volume reduction for the combined loading condition. This produces more economical topologies. In terms of the computational cost, the initial domain under combined loading has a regular mesh of 3174 elements. At the selected intermediate stage, each element of the regular mesh is further divided into 4 smaller elements, thereby generating a refined mesh of 7264 elements. The computational effort for this exercise is much less as compared to using a refined mesh of 12696 elements from the very beginning of the optimisation process.

\subsubsection{Cable-stayed bridge}

The dimension of the initial design domain for the cable-stayed bridge is $640 \mathrm{~m} \times 160$ m. The regular mesh for the combined loading, containing $116 \times 29$ square elements, is shown in Fig. 8(a) under the first nine load cases. The unit virtual load (i.e. Load case 10) is applied on top of the deck at the mid-span. The two ends of the deck as well as the bottom of the towers are assumed fixed. In cable-stayed bridges, the superstructure is suspended by cables attached directly to the towers, which together with the end supports carry the entire load of the bridge. The concrete deck acts as a part of the support system, functioning as a 
horizontal compression member. The towers may be of concrete material as well. The cables work solely in tension hence steel is normally used. The element thickness of the non-design deck and of the towers is $20 \mathrm{~m}$ and that of the design domain for cables is $1 \mathrm{~m}$. The prescribed mid-span displacement limit is $0.1 \mathrm{~m}$. The tension-dominant design algorithm is used and the $P I_{d, t}$ formula as given Eq. (12) is monitored to determine the optimum topology.

Under the combined loading condition, the regular mesh of 3364 elements is optimized until an intermediate iteration when the formation of cables begins. Subsequently, each element of the regular mesh is further divided into 4 smaller elements, thereby generating a refined mesh with 4104 elements, as shown in Fig. 8(b). The selection of the intermediate stage is indicated in Fig. 9 where $P I_{d, t}$ jumps at iteration 97 with the regular mesh scheme. The refined mesh is further optimized until iteration 242 when $P I_{d, t}$ reaches the maximum. This also corresponds to a remarkable volume reduction, or 91\%. For the two loading conditions, the optimum topologies (obtained at maximum $P I_{d, t}$ ) are shown in Fig. 10. Both topologies feature two rather thin cables for the outer spans connecting, at the towers, another two in the navigational span. The proposed scheme is far superior to the previous analysis with a regular mesh in that the new design bears a strong resemblance to the real cable-stayed bridges. Furthermore, equally satisfactory outcomes are produced without incurring substantially higher computational effort. Note that for a complete analysis, 13456 elements have to be involved at the first iteration (compared to 4104 elements used in the refined mesh scheme).

\subsubsection{Suspension bridge}

The suspension bridge under consideration has straight back stays and a central span of $1428 \mathrm{~m}$. The overall design area is $2520 \mathrm{~m} \times 300 \mathrm{~m}$ and is discretized into 3004 square elements under the combined loading, as illustrated in Fig. 11(a). Load case 10, a unit virtual load, is again located on top of the deck at the mid-span. The towers and the deck, of steel and with $35.5 \mathrm{~m}$ thickness, are taken to be non-design domains. All vertical steel hangers, of $1 \mathrm{~m}$ thick in cross-section, are also assumed non-designable. The focus of this exercise is to produce an optimum cable profile in the central span region. As such, the back stays are defined as the known conditions and hence they are also non-designable. In addition, to ensure the continuity of the main cable, eighteen elements just above the deck at the mid-span 
region are made non-design domains; they, together with the back stays and the design domain for the main cable, have steel material properties and their element thicknesses are $2 \mathrm{~m}$.

In practice, the superstructure of suspension bridges is suspended with secondary vertical hangers from the main cables, which ride across the towers and share the load with the massive anchorages at each end of the bridge. To closely simulate the real situation, the two ends of the deck as well as the anchorage bases are assumed to be roller support, and the bottoms of the towers are fixed. In addition, three nodes on the top of the tower are also restrained to reflect the higher stiffness of the tower as compared to that of the main cable. The anchorage is simulated by an inclined anchorage force (of $4500 \mathrm{kN}$ ) which is transferred through the back stays to the main cable. The prescribed mid-span displacement limit is 0.2 $\mathrm{m}$. The tension-dominant design algorithm is used and the $P I_{d, t}$ is examined to determine the optimum topology.

The refined mesh scheme is also used in this optimisation process. The refined mesh (Fig. 11(b)), under the combined loading, is formed when the $P I_{d, t}$ under the regular mesh scheme experiences a sudden jump (in this case at iteration 39). This can be seen in the optimisation history presented in Fig. 12. With the proposed refined mesh scheme, the optimum topologies (Fig. 13) are achieved at iterations 169 and 164, respectively, for the two loading conditions, and the corresponding volume reductions are $77 \%$ and $75 \%$. Similar topologies are noted for both loading conditions where a main cable is formed together with some extra inclined members in the central span. Such topology cannot be predetermined without the optimisation process. The topology is not unlike some of the existing bridges in the world, for example, the Brooklyn Bridge in New York (see Ref. [26]) where a combined system containing both suspension and stay cables is constructed. Furthermore, a much better design is achieved with the proposed scheme in terms of both topologies and computational time (see Ref. [25]).

\section{Optimisation of cable supported bridges with frequency constraints}

The optimum design of bridge structures with multiple constraints is of great importance, particularly for cable supported bridges. The deformation, the static and dynamic stabilities are the design factors necessary to be considered in such bridges. As a pilot study for dynamic stability consideration, the cable-stayed and suspension bridges 
optimised with the stress and displacement constraints are further investigated with frequency constraint. Cable supported bridges are studied because they are more sensitive to vibration than arch type bridges. In the analysis, the frequency optimisation with "nibbling" technique is performed with the aim of increasing the fundamental frequency of the bridge. Note that only the topologies generated under the combined UDL and moving loads are investigated with the frequency constraint.

\subsection{Cable-stayed bridge}

For the cable-stayed bridge under the combined loading, the optimum topology has been achieved with both stress and displacement constraints (see Fig. 10(b)). Before commencing the frequency optimisation, each element in Fig. 10(b) is further divided into 2 $\times 2$ smaller elements. This generates an even finer mesh which now becomes the initial domain. This is given in Fig. 14(a) which also shows the fundamental frequency $\omega_{1}$ of $0.69038 \mathrm{~Hz}$. The frequency optimisation with "nibbling" technique is then performed in an attempt to increase $\omega_{1}$ by altering the perimeter of the topology in Fig. 14(a). The final optimum topology satisfying the frequency constraint is presented in Fig. 14(b) which confirms that the inner cables become more slender. This is not unlike the real situation where the inner cables are generally thinner than the outer ones. The optimisation history illustrated in Fig. 15 indicates that $\omega_{1}$ is increased by $0.32 \%$ from $0.69038 \mathrm{~Hz}$ to $0.69262 \mathrm{~Hz}$. The corresponding volume reduction is $9.07 \%$, which suggests a further improved design.

\subsection{Suspension bridge}

For the suspension bridge, each element (of regular mesh) in the optimum topology produced with the stress and displacement constraints is subdivided into $3 \times 3$ smaller elements. This results in a denser mesh as the initial domain for frequency optimisation. The denser mesh is shown in Fig. 16(a) and its $\omega_{1}$ is found to be $0.21000 \mathrm{~Hz}$. The resulting optimum topology with frequency constraint is displayed in Fig. 16(b), while Fig. 17 details the corresponding optimization history. The increase in $\omega_{1}$ is $34.40 \%$ with a further volume reduction of $1.52 \%$, which indicates an improvement of the design. 


\section{Concluding remarks}

The principal stress based ESO method is employed to optimise various types of bridges. The arch, tied arch, cable-stayed and suspension bridges are selected because of their distinct structural forms. Basically they are an assembly of major or dominant tension and/or compression components. The method, in conjunction with the displacement sensitivity number, can be employed to effectively produce tension- and compressiondominant topologies while maintaining the overall structural performance. The research outcome indicates that when the compression-dominant design algorithm is adopted in the optimisation process, an arch or a tied arch bridge emerges. If, on the other hand, the tension-dominant design algorithm is used, a cable-stayed or a suspension bridge evolves. The use of the appropriate optimisation algorithm has produced most efficient design topology in terms of uniform stress and least weight for given bridge structures with certain span, loading and support conditions.

Two performance index formulas are developed to determine the optimum topologies for each of the bridge types. To improve the details of the final design, a refined mesh scheme is proposed. The numerical results reveal that the proposed scheme has led to economy in solution time without resorting to the complete analysis of a finer mesh. The frequency optimisation with "nibbling" technique is also performed to increase the fundamental frequency of cable supported bridges as well as to improve details of the topologies already generated with the stress and displacement constraints.

With various design constraints, the optimum topologies of bridge structures are produced that make the best utilisation of the available materials. The incorporation of stress, displacement and frequency constraints for cable supported bridges also attempts to address practical topology design problems associated with static and dynamic considerations. In addition, the optimum topologies, having minimum volume (weight) and maximum structural performance, bear strong resemblance to the real bridge structures. The applicability, simplicity and effectiveness of the principal stress based ESO method are validated through its satisfactory application in the optimum design of various types of bridges. The capability of the method will be further extended to consider buckling constraint to deal with the slenderness and geometric instability conditions, in particular for compression-dominant design optimisation. In addition, at its preliminary development 
stage, the current study deals with two-dimensional behaviour of bridge type structures. The extension of the proposed method to three-dimensional situations certainly merits further investigation. Furthermore, a variation of span length, overall height, loading and support conditions, as well as the positions and amount of hangers will be investigated to examine their influence on the optimum topologies. The outcome of such investigation will certainly help provide more information for preliminary design of bridge type structures.

It should also be mentioned that further work has been carried out to incorporate frequency constraint in a repeated cycle of topology and shape optimisations (see Ref. [29]) where increasing fundamental frequency together with imposing stress and displacement constraints are capable of producing a topology with more rational distribution of available materials.

The potential advancements of the sophisticated bridge topology optimisation are manifold. Through the optimum topology design, the designer can discover completely new and innovative bridges. The optimisation techniques can also be used to benchmark existing bridges as well as to design and build new bridges. Given specific geography of bridge location, construction requirements and available materials, the design engineers, with the aid of topology optimisation techniques, are able to produce more efficient, economical and feasible designs.

\section{Acknowledgments}

The support from Griffith University Research Grant scheme 1999 is gratefully acknowledged. 


\section{References}

[1] Bendsøe MP, Kikuchi N. Topology and layout optimization of discrete and continuum structures. In: Kamat MP, editor. AIAA progress in aeronautics and astronautics series, vol.150. Structural optimization - status and promise. Washington DC: AIAA, 1993.

[2] Topping BHV. Topology design of discrete structures. In: Bendsøe MP, Mota Soares CA, editors. Topology design of structures. Kluwer Academic Publishers, 1993.

[3] Rozvany GIN, Bendsøe MP, Kirsch U. Layout optimization of structures. Applied Mechanics Review ASME 1995;48(2):41-119.

[4] Thierauf G. Optimal topologies of structures: homogenization, pseudo-elastic approximation and the bubble-method. Engineering Computation 1996;13(1):86-102.

[5] Achtziger W. Truss topology optimization including bar properties different for tension and compression. Structural Optimization 1996;12:63-74.

[6] Rozvany GIN. Structural design via optimality criteria. Dordrecht: Kluwer Academic, 1989.

[7] Zhou M, Rozvany GIN. DCOC: an optimality criteria method for large systems. Part I: theory. Structural Optimization 1992;5:12-25.

[8] Rozvany GIN. Topology optimisation of multipurpose structures. In: Steven GP, Querin OM, Guan H, Xie YM, editors. Proc. Australasian Conf on Structural Optimisation. Australia: Oxbridge Press, 1998.

[9] Bendsøe MP, Kikuchi N. Generating optimal topology in structural design using a homogenisation method. Computer Methods in Applied Mechanics and Engineering 1988;71:197-224.

[10] Suzuki K, Kikuchi N. A homogenisation method for shape and topology optimization. Computer Methods in Applied Mechanics and Engineering 1991;93:291-318.

[11] Bendsøe MP, Diaz AR, Lipton R, Taylor JE. Optimal design of material properties and material distribution for multiple loading conditions. Int J Numerical Methods in Engineering 1995;38(7):1149-1170.

[12] Allaire G, Kohn RV. Topology optimisation and optimal shape using homogenisation. In: Bendsøe MP, Mota Soares CA, editors. Topology design of structures. Kluwer Academic Publishers, 1993.

[13] Jiang T, Papalambros PY. Optimal structural topology design using the homogenization method with multiple constraints. Engineering Optimization 1996;27:87-108. 
[14] Mlejnek HP, Schirrmacher R. An engineer's approach to optimal material distribution and shape finding. Computer Methods in Applied Mechanics and Engineering 1993;106:1-26.

[15] Goldberg DE. Genetic algorithms in search, optimisation, and machine learning. Canada: Addison-Wesley Publishing Company, 1989.

[16] Xie YM, Steven GP. A simple evolutionary procedure for structural optimization. Computers and Structures 1993;49(5):885-896.

[17] Xie YM, Steven GP. Evolutionary structural optimisation. Berlin: Springer-Verlag, 1997.

[18] Hinton E, Sienz J. Fully stressed topological design of structures using an evolutionary procedure. Engineering Computation 1995;12:229-244.

[19] Guan H, Steven GP, Querin OM, Xie YM. Optimisation of bridge deck positioning by the evolutionary method. Structural Engineering and Mechanics - An International Journal 1999;7(6):551-559.

[20] Guan H, Xue Q, Steven GP, Xie YM. Optimization of cable-stayed and tied arch bridges." In: Proc. $7^{\text {th }}$ AIAA/USAF/NASA/ISSMO Symposium on Multidisciplinary Analysis and Optimization. AIAA, 1998.

[21] Guan H, Steven GP, Xie YM. Evolutionary structural optimisation incorporating tension and compression materials. Advances in Structural Engineering - An Int J 1999;2(4):273-288.

[22] Querin OM, Steven GP, Xie YM. Evolutionary structural optimization using a bidirectional algorithm. Engineering Computations 1998;15:1031-1048.

[23] Liang QQ, Xie YM, Steven GP. Optimal selection of topologies for the minimumweight design of continuum structures with stress constraints. Proc. Inst of Mechanical Engineers. Part C: J Mechanical Engineering Science 1999;213(C8):755-762.

[24] Kirsch U. Optimal design based on approximate scaling. J Structural Engineering 1982;108(ST4):888-909.

[25] Chen YJ. Topology optimisation of bridge type structures with multiple constraints. MPhil Thesis, School of Engineering, Griffith University Gold Coast Campus, Gold Coast, Australia, 2000.

[26] Transportation Research Board. Bridge aesthetics around the world. Washington DC: Transportation Research Board, National Research Council, 1991.

[27] Xanthakos PP. Theory and design of bridges. New York: Wiley, 1994. 
[28] AustRoads. '92 Austroads bridge design code. NSW: Australasian Railway Association, 1992.

[29] McLean DJ, Guan H. Topology and shape optimisation of cable supported bridges. In: Loo YC, Chowdhury SH, Fragomeni S, editors. Proc. $17^{\text {th }}$ Australasian Conference on the Mechanics of Structures and Materials: Balkema, 2002. 


\section{List of figure captions}

Fig. 1. Initial design domain for arch bridge under UDL: (a) Load case 1; (b) Load case 2

Fig. 2. Initial design domain for arch bridge under combined UDL and moving loads: (a) Load cases 1 to 9; (b) Load case 10

Fig. 3. Optimization history of arch bridge: (a) UDL; (b) Combined UDL and moving loads

Fig. 4. Optimum topologies of arch bridge: (a) UDL; (b) Combined UDL and moving loads

Fig. 5. Design domain for tied arch bridge under combined UDL and moving loads: (a) Initial; (b) Refined

Fig. 6. Optimization history of tied arch bridge under combined UDL and moving loads: (a) Performance index; (b) Volume variation

Fig. 7. Optimum topologies of tied arch bridge with refined mesh scheme: (a) UDL; (b) Combined UDL and moving loads

Fig. 8. Design domain for cable-stayed bridge under combined UDL and moving loads: (a) Initial; (b) Refined

Fig. 9. Optimization history of cable-stayed bridge under combined UDL and moving loads: (a) Performance index; (b) Volume variation

Fig. 10. Optimum topologies of cable-stayed bridge with refined mesh scheme: (a) UDL; (b) Combined UDL and moving loads

Fig. 11. Design domain for suspension bridge under combined UDL and moving loads: (a) Initial; (b) Refined

Fig. 12. Optimization history of suspension bridge under combined UDL and moving loads: (a) Performance index; (b) Volume variation

Fig. 13. Optimum topologies of suspension bridge with refined mesh scheme (a) UDL; (b) Combined UDL and moving loads

Fig. 14. Frequency optimization of cable-stayed bridge: (a) Initial domain $\left(\omega_{1}=0.69038\right.$ $\mathrm{Hz})$; (b) Final optimum topology $\left(\omega_{1}=0.69262 \mathrm{~Hz}\right)$

Fig. 15. Optimization history of cable-stayed bridge with frequency constraint

Fig. 16. Frequency optimization of suspension bridge: (a) Initial domain ( $\omega_{1}=0.21000$ $\mathrm{Hz})$; (b) Final optimum topology ( $\omega_{1}=0.28223 \mathrm{~Hz}$ ) 
Fig. 17. Optimization history of suspension bridge with frequency constraint 


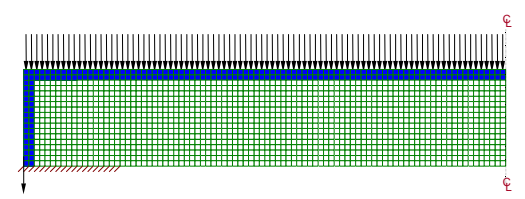

(a) Load case 1

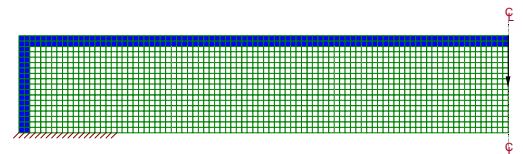

(b) Load case 2

Fig. 1

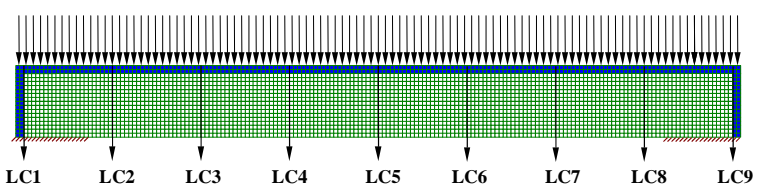

(a) Load cases 1 to 9

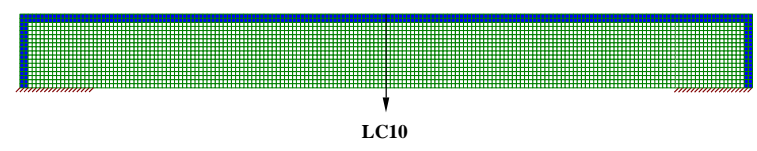

(b) Load case 10

Fig. 2

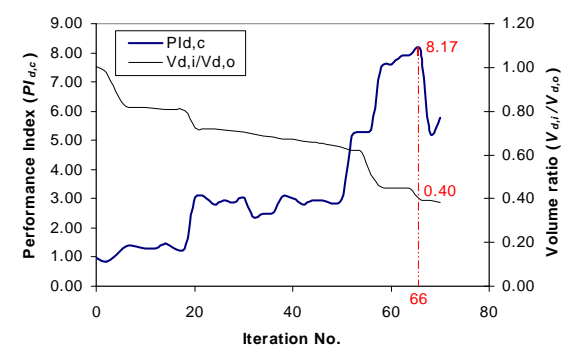

(a) UDL

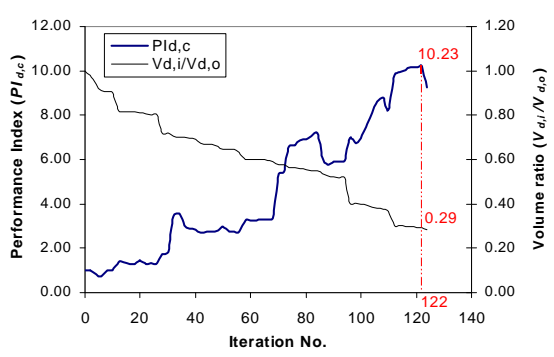

(b) Combined UDL and moving loads

Fig. 3

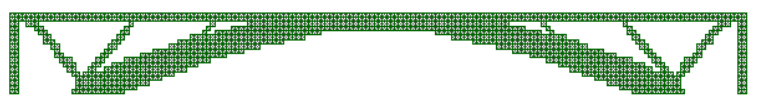

(a) UDL

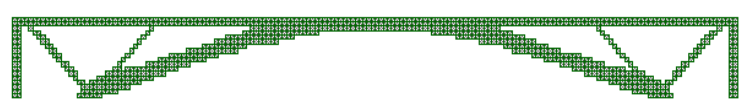

(b) Combined UDL and moving loads

Fig. 4

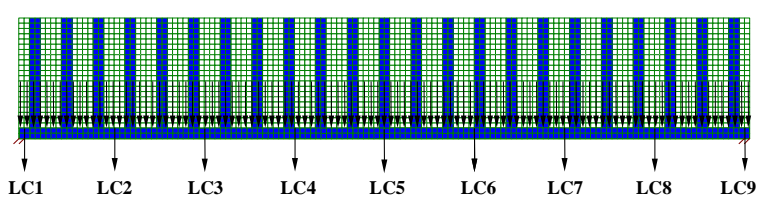

(a) Initial

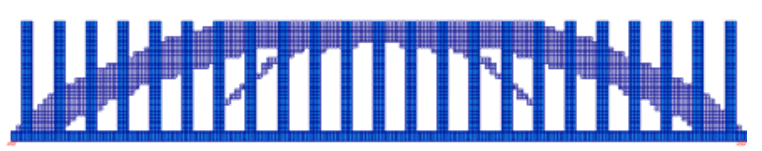

(b) Refined

Fig. 5 


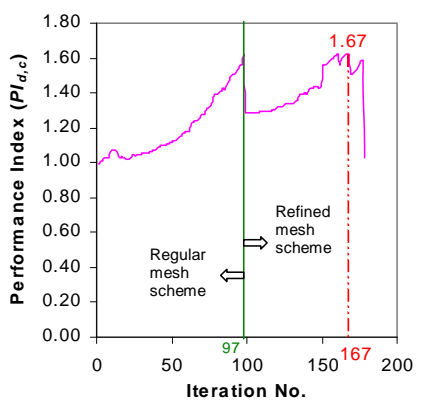

(a) Performance index

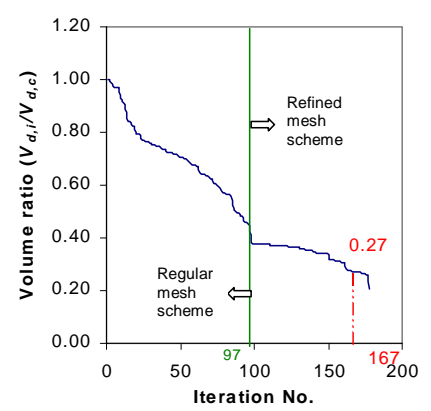

(b) Volume variation

Fig. 6

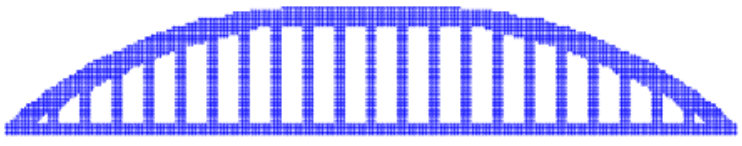

(a) UDL

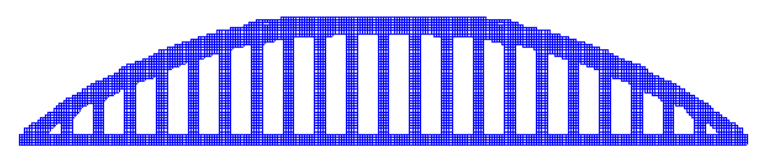

(b) Combined UDL and moving loads

Fig. 7

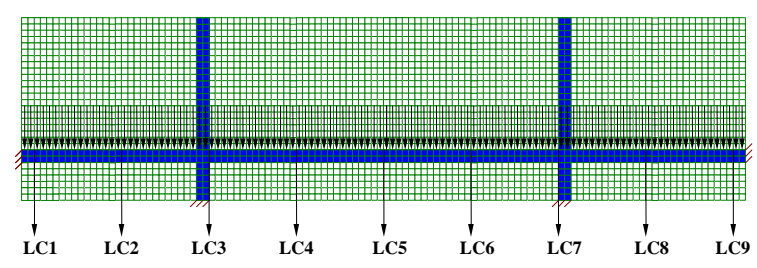

(a) Initial

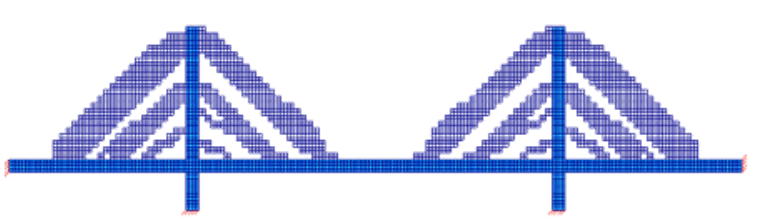

(b) Refined

Fig. 8

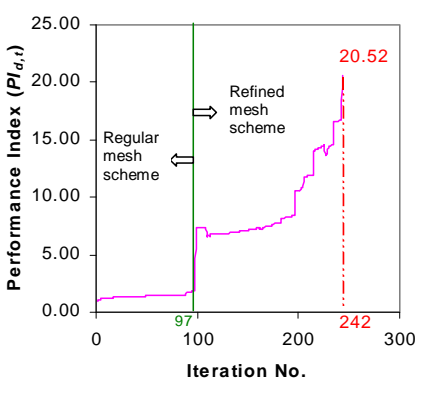

(a) Performance index

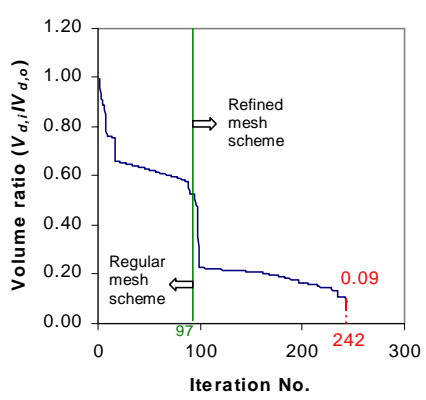

(b) Volume variation

Fig. 9

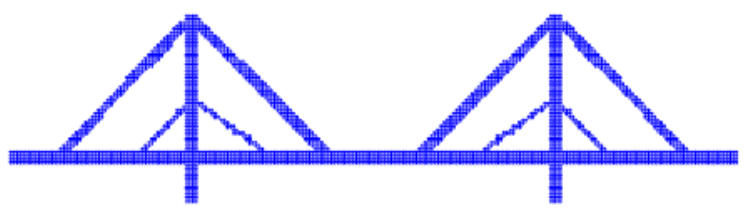

(a) UDL 


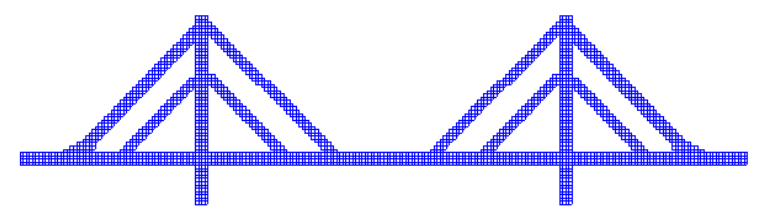

(b) Combined UDL and moving loads

Fig. 10

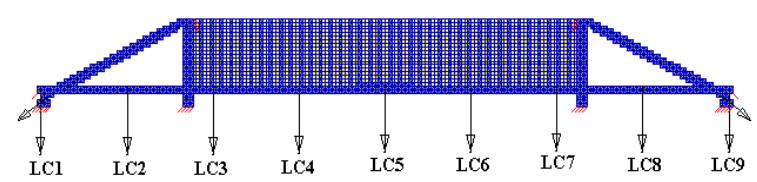

(a) Initial

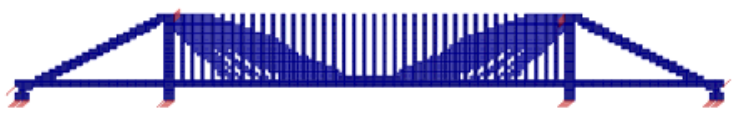

(b) Refined

Fig. 11

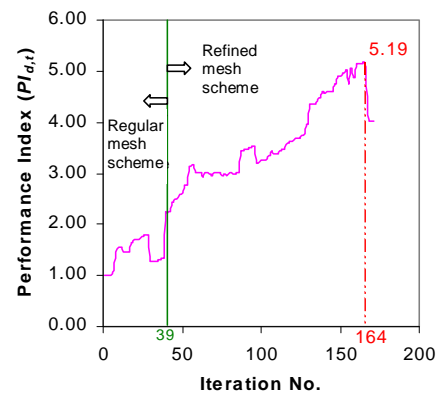

(a) Performance index

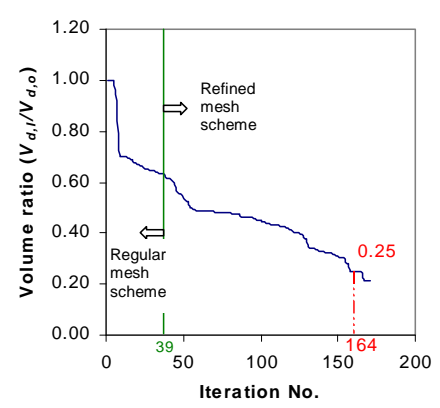

(b) Volume variation

Fig. 12

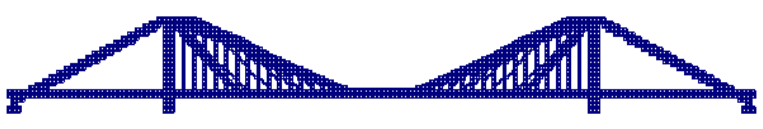

(a) UDL

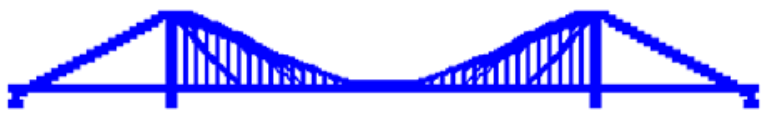

(b) Combined UDL and moving loads

Fig. 13

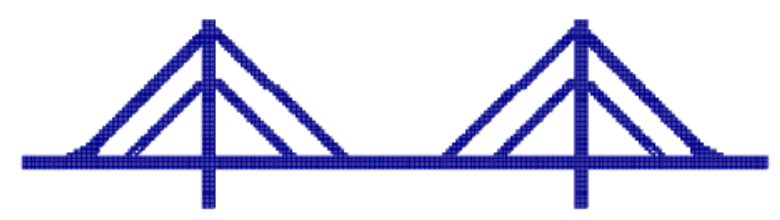

(a) Initial domain $\left(\omega_{1}=0.69038 \mathrm{~Hz}\right)$

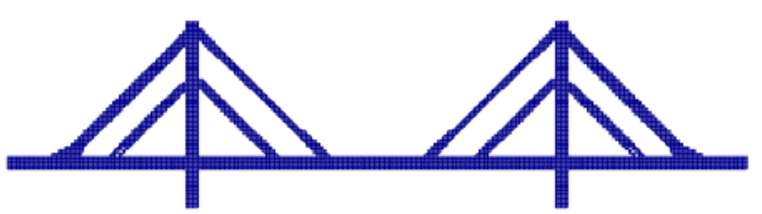

(b) Final optimum topology $\left(\omega_{1}=0.69262 \mathrm{~Hz}\right)$

Fig. 14

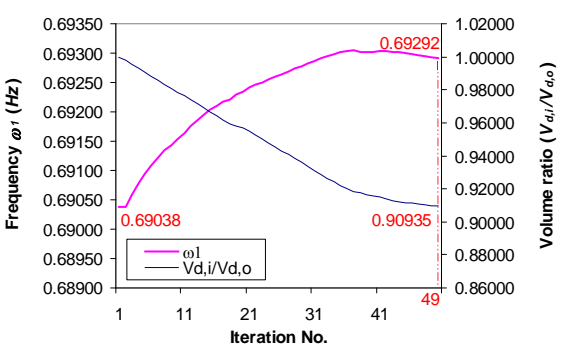

Fig. 15 


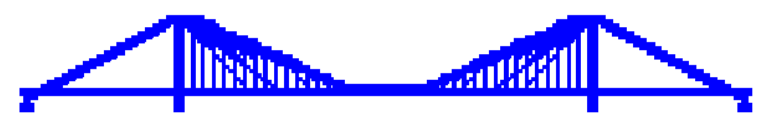

(a) Initial domain $\left(\omega_{1}=0.21000 \mathrm{~Hz}\right)$

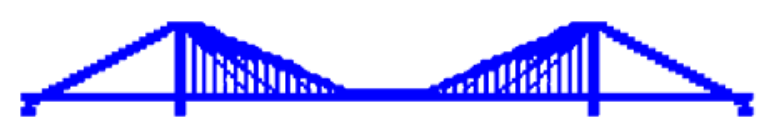

(b) Final optimum topology $\left(\omega_{1}=0.28223 \mathrm{~Hz}\right)$

Fig. 16

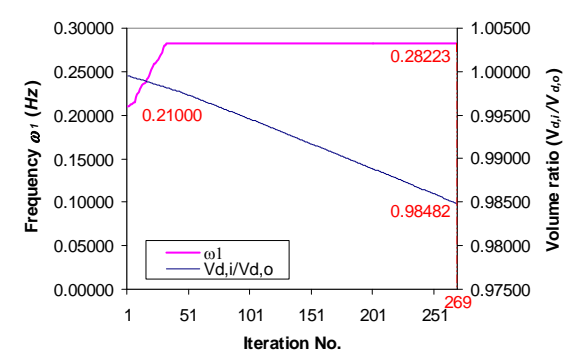

Fig. 17 